\title{
Levitation and motion of a magnet partially immersed into a magnetic fluid
}

\author{
Daria Pelevina*, Mikhail Kobzev, Sergey Kalmykov, Dmitrii Merkulov and Vera Naletova \\ Lomonosov Moscow State University, 119991, Moscow, Russia
}

\begin{abstract}
The levitation of a permanent disc magnet partially immersed into a magnetic fluid volume on a horizontal plane is studied theoretically and experimentally. Motion of the magnet along a thin magnetic fluid layer is investigated experimentally. A mathematical model of the magnet dynamics is proposed.
\end{abstract}

\section{Introduction}

In [1] static levitation of a magnet inside a magnetic fluid (MF) in a cylindrical vessel on a horizontal plane is observed for the first time. The motion of a magnet along a thin MF layer is studied experimentally in $[2,3]$. The mathematical model of the magnet motion is not proposed in $[2,3]$. In the present paper, static levitation of a disc magnet partially immersed into a MF with free surface (without a vessel in contrast to [1]) on a horizontal plane is studied theoretically and experimentally. The motion of a disc-like magnet along a MF layer on a horizontal plane is studied experimentally. The influence of the layer thickness and length and of the MF volume around the magnet on the magnet trajectory is studied experimentally, in contrast to $[2,3]$. A mathematical model of the magnet dynamics is proposed. The comparison of experimental and theoretical data allows us to estimate the friction coefficient and the height of the MF layer remaining behind the magnet.

\section{Statics}

\subsection{Experiment}

In the MF volume, a magnet can levitate in equilibrium, as it is shown, e.g., in [1], at a certain distance $h$ from the plane (levitation height).

Fig. 1. Scheme of the magnet immersed into a MF volume.

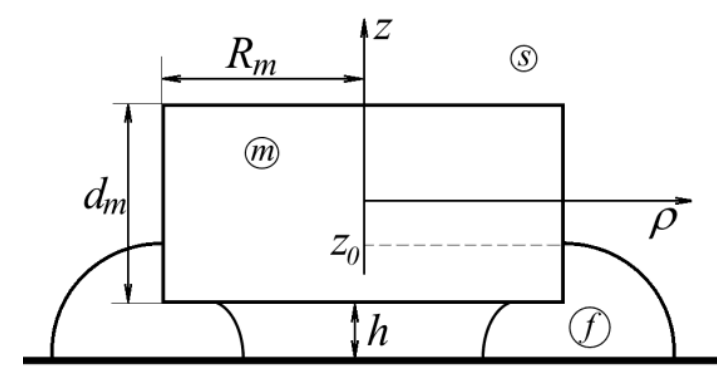

\footnotetext{
* Corresponding author: pelevina.daria@gmail.com
}

We introduce a cylindrical coordinate system with the centre at the magnet centre, and the $z$-axis is directed vertically upward (see Fig. 1). In the experiments, a cylindrical (disc-like) magnet made of $\mathrm{NdFeB}$ rare earth alloy (height $d_{m}=2.04 \mathrm{~mm}$, radius $R_{m}=3 \mathrm{~mm}$, $m_{m}=0.4 \cdot 10^{-3} \mathrm{~kg}$ ), see Fig. 1 , is used. The magnetic field intensity $H_{0}$ of the magnet is measured at the distance of $1 \mathrm{~mm}$ from the magnet surface at center $(\rho=0$, $\left.z=d_{m} / 2+1 \mathrm{~mm}\right)$ by the Hall sensor $H_{0 c}=187 \mathrm{kA} / \mathrm{m}$. The MF (Ferrotec) based on a light hydrocarbon (initial susceptibility $\chi_{0 f}=1.89 \quad\left(\mu_{f}=1+\chi_{0 f}\right), \quad$ saturation magnetization $M_{f}=31.8 \mathrm{kA} / \mathrm{m}$, density $\rho_{f}=1210 \mathrm{~kg} / \mathrm{m}^{3}$, viscosity $\eta_{f}=6 \mathrm{mPa} \cdot \mathrm{s}$ ) is chosen. The environment (air, NMF) is not magnetizable $\left(M_{s}=0\right)$ and its density is small in comparison with the MF density $\left(\rho_{s}=1.2 \mathrm{~kg} / \mathrm{m}^{3}\right.$ $\left.<<\rho_{f}\right)$. Here and after, the parameters corresponding to the magnet, the MF and the NMF are noted by the indices $m, f$, $s$, respectively.

Fig. 2. The MF shape around the disc magnet:

1 - experiment, side view; 2 - experiment, bottom view;

3 - theory; $\mathrm{a}-V=1 \mathrm{~mm}^{3} ; \mathrm{b}-V=2.9 \mathrm{~mm}^{3}$; $\mathrm{c}-V=36.7 \mathrm{~mm}^{3}$.
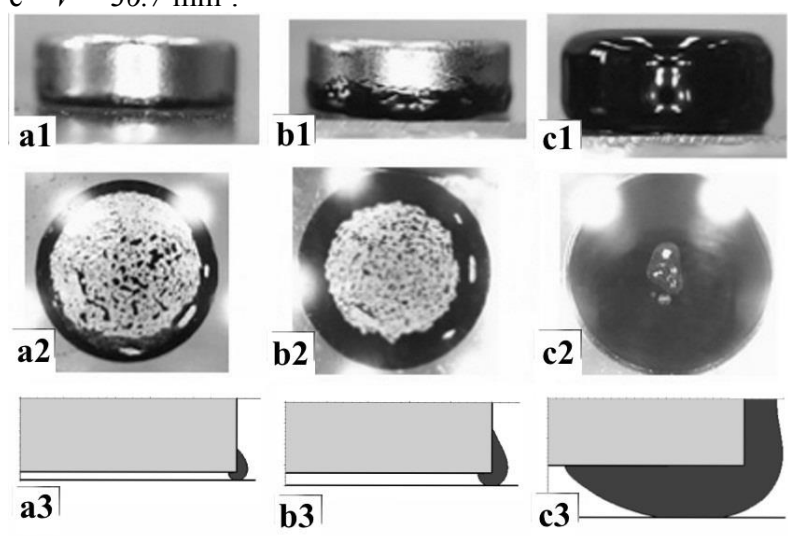

The magnet is placed in the MF volume on a horizontal plane. The magnet collects the MF around itself, so the MF has a symmetrical surface shape with respect to the $z$-axis, see Fig. 2, row 1 . It is 
experimentally observed that the magnet can levitate in the MF volume at the height $h$. Different MF volumes are considered. The limited MF volume $\left(V<4.26 \mathrm{~mm}^{3}\right)$ corresponds to the vertical coordinate of the contact between the MF surface and the lateral surface of magnet $z_{0}<0$. For a small MF volume $V<3.2 \mathrm{~mm}^{3}$ $\left(z_{0}<-0.33 \mathrm{~mm}\right)$ the MF touches only the lower part of the magnet. For big volumes, the MF also touches the upper part of the magnet. There is a region not occupied by the MF under the magnet, see Fig. 2, row 2.

The levitation height $h$ for different MF volumes is measured in the experiment, see Fig. 3, points. The levitation height $h$ increases with the increasing of the MF volume.

Fig. 3. The dependence of the levitation height $h$ on the MF volume $V$.

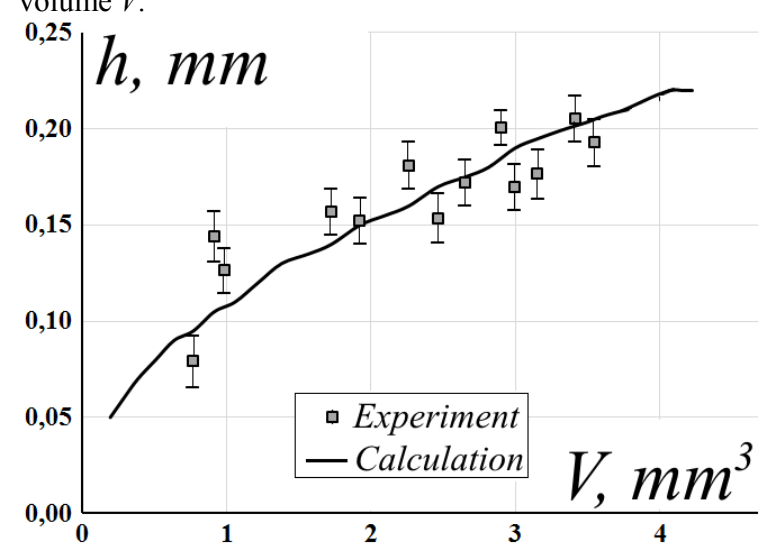

\subsection{Theory}

Let us consider the statics of a disc magnet in a small MF volume on a horizontal plane under the following assumptions: 1) Non-induction approximation: $\mu_{f}-$ $1<<1\left(\mu_{f}=\right.$ const $)$. In this case, we can assume that the magnetic field is a magnet field in the vacuum $H \approx H_{0}$ (the distortion of the magnetic field by the MF shape is not considered). 2) There is no surface tension $(\sigma=0)$ at the boundary MF - NMF. 3) We neglect the influence of gravity on the MF shape. These assumptions are true near the magnet, since the magnetic force is much greater than the forces of gravity and of surface tension.

The stress tensor has the form $\left(k=f, s ; \boldsymbol{B}=\mu_{0} \mu_{f} \boldsymbol{H}\right)$ :

$$
p_{i j}^{(k)}=-p_{k} g_{i j}+\tau_{i j}^{(k)}, \tau_{i j}^{(k)}=H_{i} B_{j}-\frac{H B}{2} g_{i j}
$$

It follows from the equilibrium equation that the pressures in the MF and the NMF are constant: $p_{s}=$ const,$p_{f}=$ const . The dynamic boundary condition on the interface MF - NMF (which is described by the equation $z=f(\rho))$ in the assumptions described above has the following form:

$$
0=p_{n n}^{(s)}-p_{n n}^{(f)} \approx p_{f}-p_{s}+\mu_{0}\left(\mu_{f}-1\right) \frac{H_{0 f}^{2}}{2}
$$

Here $\boldsymbol{n}$ is the external normal to the MF, $H_{0 f}$ is the magnetic field modulus on the MF surface. It follows from (2) that the MF surface coincides with the curves of constant magnetic field values: $H_{0 f}(\rho, z)=$ const. We can express the surface force acting on the magnet, taking into account $\nabla^{j} \tau_{i j}^{(k)} \mathbf{e}_{\mathbf{i}}=-H^{2} \mu_{0} \nabla \mu_{k} / 2=0$ and that the magnetic field decreases as $o\left(1 / r^{2}\right)\left(r^{2}=z^{2}+\rho^{2}\right)$, as follows:

$$
\mathbf{F}=\int_{S_{m}} p_{i j} n_{m}^{j} e^{i} d s=\mu_{0} \frac{\left(\mu_{f}-1\right)}{2} \int_{S_{b}}\left(H^{2}-H_{0 f}^{2}\right) d s \mathbf{e}_{\mathbf{z}}
$$

Here $\boldsymbol{n}_{\boldsymbol{m}}$ is the external normal to the magnet, $S_{m}$ is the surface of the magnet, $S_{b}$ is the contact surface between the MF and the plane. The magnetic field $H_{0}$ is simulated using the ANSYS software package for parameters mentioned above.

The equilibrium equation for the magnet has the following form:

$$
\begin{aligned}
& \mathbf{F}+\mathbf{P}+\mathbf{P}_{A}=0, \mathbf{P}=m_{m} \mathbf{g}, \\
& \mathbf{P}_{A}=-\left(d_{m}+z_{0}\right) S_{m f} \rho_{f} \mathbf{g}
\end{aligned}
$$

Here $S_{m f}$ is the area of the magnet bottom wetted by the MF. For a given MF volume $V$ from (2) and (4) the MF shape and the levitation height $h$ can be determined by the following way. Let the MF surface $z=f(\rho)$ begins at some point with coordinates $\left(\rho_{0}, z_{0}\right)$. We can calculate the magnetic field modulus at this point: $H_{0 f}=H\left(\rho_{0}, z_{0}\right)$. From the equation $H(\rho, z)=H_{0 f}$, using Maple software, we compute the MF shape $z=f(\rho)$ up to the intersection with the horizontal plane $z=-d_{m} / 2-h$. The value of the height $h$ is chosen in a manner that the equilibrium condition (4) is satisfied. Then the MF volume $V$ is calculated.

Calculated MF shapes, obtained for the experimental data, are shown in Fig. 2, row 3. Only the lower right quadrant is shown in Fig. 2, row 3 because of the symmetry. The curve in Fig. 3 shows the dependence of the levitation height $h$ on the MF volume $V$. Fig. 3 shows that the results of numerical calculations and of experimental studies are in a good agreement.

It should be noted that in $[2,3]$ the study of the magnet statics in the MF is not carried out.

\section{Dynamics}

If the cylindrical magnet is immersed in the nonsymmetric MF volume, horizontal force acting on a levitated magnet arises. Under the influence of this force, the magnet can move along the plane. The motion of a magnet, partially immersed in the MF, along a thin MF layer, see Fig. 4, is investigated experimentally and theoretically.

\subsection{Experiment}

The magnet is placed on the left edge of the MF layer (thickness $d_{l}$, length $l_{l}$, height $h_{l l}=0.3 \mathrm{~mm}$ ), see Fig. 8 . In $[2,3]$, the layer is a trace of the magnet sliding down the inclined plane. In contrast, here we fill a rectangular region of a given size by the MF using a syringe to make a layer. Parameters of the layer are denoted by the index $l$. The magnet levitates and moves to the right, collecting the MF. Thus, the MF non-symmetry with respect to the magnet centre remains until the magnet reaches the right layer edge: the MF amount on the right side of the magnet is greater than on the left side. For some 
parameters of the experiment, the magnet does not collect all the MF in the layer and the MF layer, which is thinner than the initial layer, remains on the left side of the magnet. Height of this layer is $h_{l 2}<h_{l 1}$. In this case, when the magnet reaches the right edge, there is a nonsymmetry of the MF volume with respect to the magnet centre: the MF amount on the left side is more than on the right side. Therefore, the magnet moves to the left and can return to its initial position, see Fig. 4.

Fig. 4. The magnet motion in a MF layer in experiment $\left(d_{l}=\right.$ $4 \mathrm{~mm}, l_{l}=160 \mathrm{~mm}$ ), top view. Time between frames $t=1 / 3 \mathrm{~s}$.

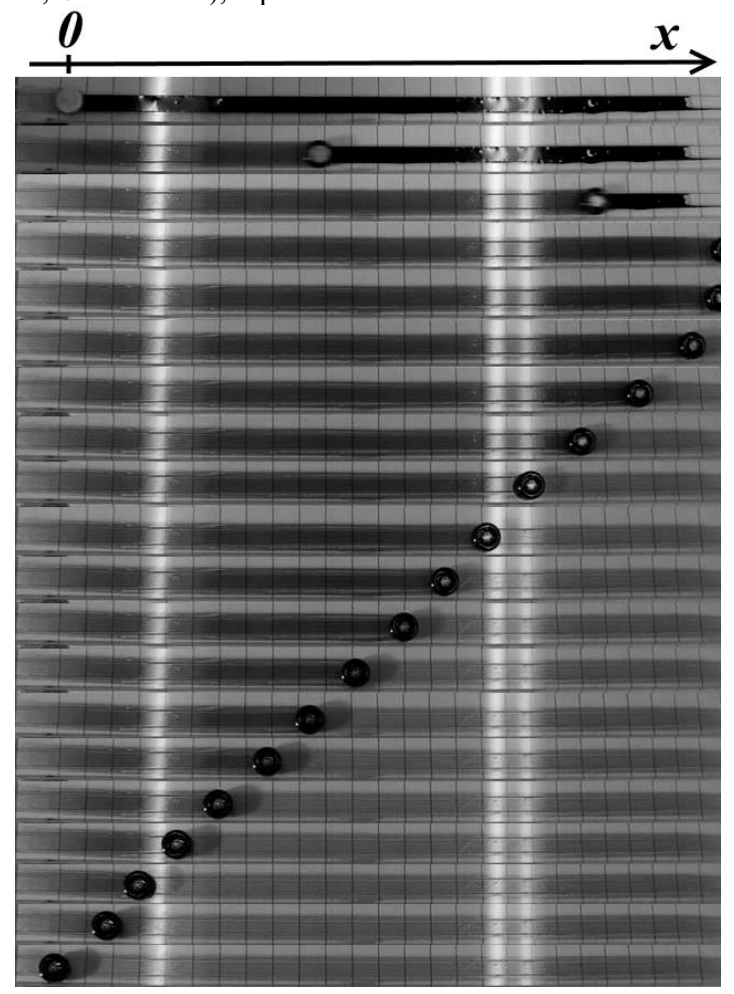

The dependencies of the position of the magnet on time, using numerical processing of experimental video, are plotted. Different thicknesses $d_{l}$, lengths $l_{l}$ of the MF layer, and the MF volumes $V$ on the magnet at the initial position $(t=0)$ are considered.

Fig. 5. The dependencies of the magnet position $x$ on time $t$ for $d_{l}=3 \mathrm{~mm}, h_{l l}=0.3 \mathrm{~mm}, V=14.5 \mathrm{~mm}^{3}$ and for different layer lengths: $1-l_{l}=50 \mathrm{~mm} ; 2-l_{l}=100 \mathrm{~mm} ; 3-l_{l}=150 \mathrm{~mm}$.

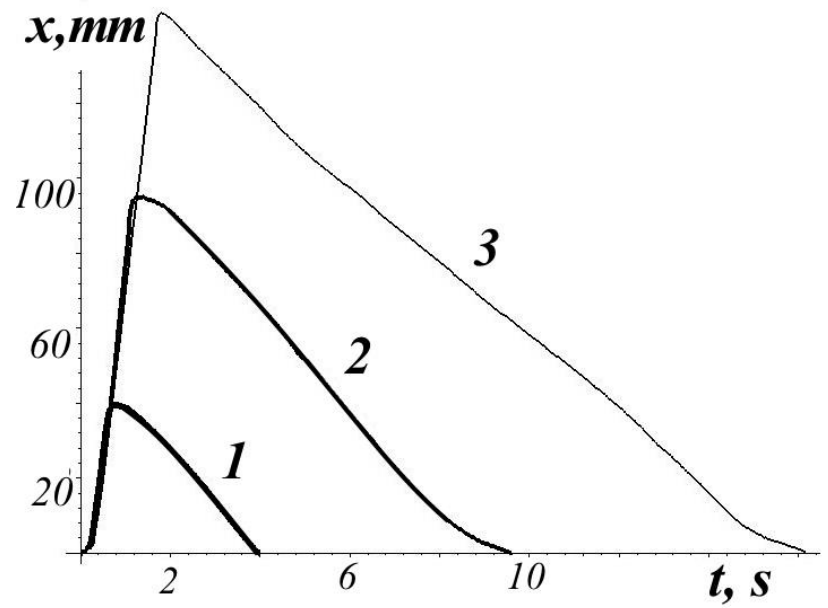

For example, the dependencies of the magnet centre position $x$ on time $t$ for different layer lengths $l_{l}$ are shown in Fig. 5. The magnet is fully immersed into the $\mathrm{MF}$, as in Fig. 2, c. It is observed that far from the layer edges, the magnet moves with the nearly constant velocity $v$. The velocity $v_{l}$, when magnet moves to the right, is greater than the velocity $v_{2}$, when it moves to the left (the return motion along a thin layer $h_{12}$ ). Far from the layer edges, the constant velocities do not depend on the layer length $l_{l}$.

Fig. 6. The dependencies of the magnet centre position $x$ on time $t$ for $l_{l}=100 \mathrm{~mm}, V=0$ and for different layer thicknesses: $1-d_{l}=3 \mathrm{~mm} ; 2-d_{l}=4 \mathrm{~mm} ; 3-d_{l}=5 \mathrm{~mm}$.

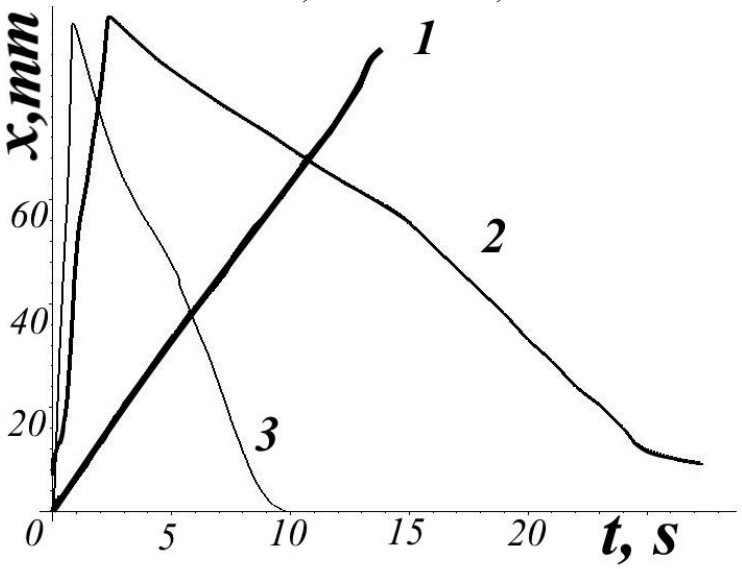

The dependencies of the position of the dry magnet $(V=0)$ on time $t$ for different layer thicknesses $d_{l}$ are shown in Fig. 6. The dry magnet collects the MF from the layer, rises above the plane and moves along the layer. Thus, in the experiment, $h$ varies significantly, for example, $h$ varies from 0 to $0.2 \mathrm{~mm}$ for curve 1 in Fig. 6 . For small thickness $d_{l}=3 \mathrm{~mm}$, the magnet has a relatively small velocity $v_{l}$ and collects all the MF from the layer, so that it does not return to its initial position. For $d_{l}=4 \mathrm{~mm}$ and $5 \mathrm{~mm}$ the magnet velocity is higher, and the magnet moves back. The velocities $v_{1}$ and $v_{2}$ increase with the increasing of the layer thickness $d_{l}$ due to the increase of the levitation height $h$ (decrease of the viscosity force) and of the magnetic force, which is proportional to the area of the edge layer section.

Fig. 7. The dependencies of the magnet centre position $x$ on time $t$ for $l_{l}=100 \mathrm{~mm}, d_{l}=3 \mathrm{~mm}$ and different MF volumes: $1-V=0 ; 2-V=2.9 \mathrm{~mm}^{3} ; 3-V=36.7 \mathrm{~mm}^{3}$.

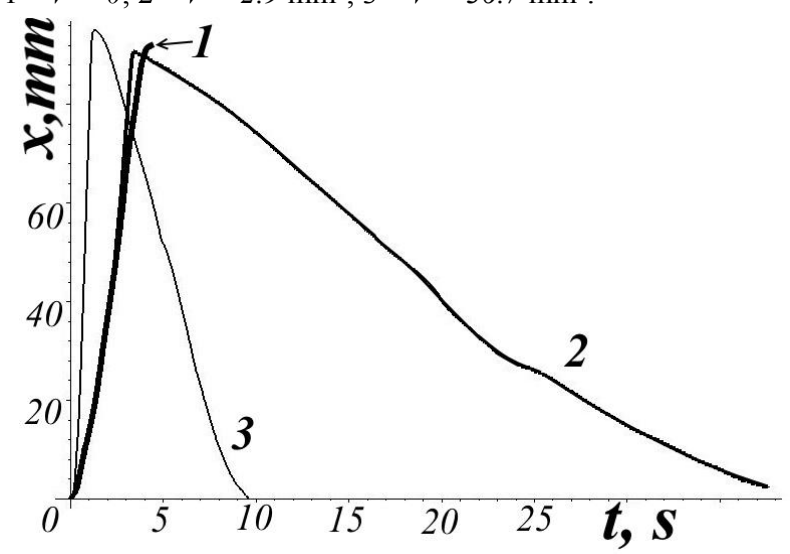


In Fig. 7, different MF volumes $V$ on the magnet at the initial position are considered. The dry magnet $(V=$ 0 , curve 1) moves more slowly, collects all the MF from the layer and does not move back. A partially immersed magnet (as in Fig. 2, b) and a fully immersed magnet (as in Fig. 2, c) have a reverse motion. Motion velocities increase with the increasing of the MF volume $V$.

In [3] an experimental investigation of a magnet dynamics is carried out for various angles of plane inclinations. In contrast, in this paper a horizontal plane is considered, but the effect of the layer parameters and of the volume $V$ on the problem is studied.

\subsection{Theory}

Let us consider the interaction between a magnet levitating in the MF volume $V$ and a MF layer with the shape of a rectangular parallelepiped, see Fig. 8. The left edge of the layer is located at the distance $r_{f}\left(r_{f}\right.$ is the radius of the MF volume) from the magnet centre. We assume that the contact surface shape between the MF volume and the MF layer is close to the rectangular shape $S_{l c}$. We introduce the fixed $x$-axis horizontally along the layer. The beginning of the $x$-axis is in the magnet centre at its initial position. If the magnet completely collects all the MF from the layer, the expression for the horizontal component of the force acting on the magnet at rest from the MF layer with the surface $S_{l}$ is written as following:

$$
F_{x 1}=-\int_{S_{l}} p_{x j} n_{l}^{j} d s=\mu_{0} \frac{\mu_{f}-1}{2}\left(\int_{S_{l c}} H^{2} d s-\int_{S_{l f}} H^{2} d s\right)>0
$$

Here $S_{l c}$ and $S_{l f}$ are the left and right edges of the layer (see Fig. 6). If the MF layer of the thickness $h_{l 2}$ remains to the left of the magnet, then the force $F_{x 2}<0$, acting from the left layer, is calculated in a similar way to (5).

Fig. 8. The interaction between the magnet and the MF layer.

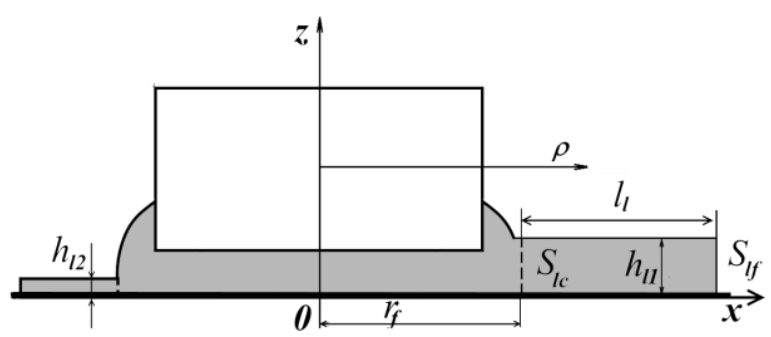

To calculate the magnet motion, we assume that the levitation height $h$ and the MF shape do not change during the motion. We also assume that the viscous force has the form $\boldsymbol{F}_{f r}=-\mathrm{k} \boldsymbol{v}, \boldsymbol{v}$ is the magnet velocity, $k=$ const.

The magnet motion under the action of the force $F_{x}=F_{x l}+F_{x 2}$ and of the viscous force $F_{f r}$ is described by the following equation:

$\left(m_{m}+m_{f}\right) \ddot{x}=F_{x}(x)-k \dot{x}, \dot{x}(0)=0, x(0)=0$

Taking into account that far from the layer edges, in the non-inertial region during the motion to the right and to the left, the velocity is constant, we can write:

$$
k v_{1}=F_{x 1, \text { max }}+F_{x 2 \text {, max }}, \quad k v_{2}=F_{x 2 \text {, max }}
$$

Here $F_{x l, \max }$ and $F_{x 2, \max }$ are the maximum values of the magnetic force from right and left layers, $v_{l}$ is the velocity, when the magnet moves to the right, $v_{2}-$ to the left. The parameters of the MF right layer are measured in the experiment, so we can calculate the magnetic force $F_{x l}$ and find the maximum force $F_{x 1, \max }$. The velocities $v_{l}$ and $v_{2}$ are known from the experiment. Under the assumption that $F_{x i, \max } \sim h_{l i}(i=1,2)$, from (7) we can find the coefficient $k$, the force $F_{x 2 \text {, max }}$ and the thickness of the layer $h_{l 2}$ remaining behind the magnet.

For the following parameter values, corresponding to the experiment, see Fig.5, curve 1: $V=14.5 \mathrm{~mm}^{3}$, $h=0.6 \mathrm{~mm}, x_{0}=r_{f}=3.8 \mathrm{~mm}, d_{l}=3 \mathrm{~mm}, h_{l l}=0.3 \mathrm{~mm}$, $l_{l}=50 \mathrm{~mm}, v_{1}=88.8 \mathrm{~mm} / \mathrm{s}, v_{2}=-13.3 \mathrm{~mm} / \mathrm{s}$, we obtain: $k=46 \mathrm{~g} / \mathrm{s},\left|\mathrm{F}_{\mathrm{x} 2 \max }\right| / \mathrm{F}_{\mathrm{x} 1 \max } \mid=0.13, h_{l 2}=0.039 \mathrm{~mm}$.

\section{Conclusion}

The statics of a permanent disc-like magnet in a MF volume on a horizontal plane is experimentally and theoretically investigated. The analytical expression for the force acting on the magnet from the small surrounding MF volume is obtained in the static case in the non-inductive approximation. The magnetic field of the magnet is modelled in ANSYS. Various static shapes of the MF surface are calculated and obtained in the experiments. The dependence of the levitation height of the magnet on the MF volume is investigated. A good coincidence between the theoretical and experimental results is obtained.

The motion of a magnet along a MF layer on a horizontal plane is experimentally studied for various initial conditions (the MF layer geometry, the MF volume on the magnet). It is shown that the increase of the MF volume and of the layer thickness leads to the increase of the magnet velocity. It is found that for some parameters the magnet returns to its initial position. A mathematical model of the magnet dynamics along a thin MF layer is proposed. The expression for the force acting on the magnet from the MF layer is obtained analytically. In the non-inertial approximation, the estimations for the viscous force coefficient and the height of the layer to the left of the magnet are obtained from the comparison with the experiment.

This study can be used to create new types of actuators based on a MF.

This work was supported by the RFBR grant 16-31-60091 and partially supported by FASIE $8541 \Gamma \mathrm{V} / 2015$.

\section{References}

1. R. E. Rosensweig, Nature, 210, 5036, p. 613 (1966)

2. Hartung S., Rehberg I., Richter R., Abs. Book: 14th German Ferrofluid Workshop, 82 (2014)

3. Freundorfer A., Rehberg I., Richter R., Abs. Book: 15th German Ferrofluid Workshop, 74 (2015) 